\title{
Perception of the Community Toward Transition of Pharmaceutical Care Services from Ministry of Health Primary Healthcare Centers to Community Pharmacies
}

Noha Al Aloola ( $\square$ nalaloola@ksu.edu.sa )

King Saud University College of Pharmacy https://orcid.org/0000-0001-9666-5426

Sumaiah Aljudaib

Riyadh Elm University

Fathy Behery

Riyadh Elm University

Monira Alwhaibi

King Saud University College of Pharmacy

Tariq Alhawassi

King Saud University College of Pharmacy

\section{Research article}

Keywords: Wasfaty, Community Pharmacy, Pharmaceutical Care Services, Saudi community

Posted Date: December 15th, 2020

DOI: https://doi.org/10.21203/rs.3.rs-124402/v1

License: (c) (1) This work is licensed under a Creative Commons Attribution 4.0 International License.

Read Full License 


\section{Abstract}

Background: Pharmaceutical care services in Saudi Arabia recently transferred from Ministry of Health $(\mathrm{MOH})$ primary healthcare centers (PHCs) to community pharmacies (Wasfaty service). However, there is a lack of research on the need and impact of this transition. This study explored the Saudi community's perception toward the transition of pharmaceutical care service from MOH PHCs to the Wasfaty service. Community needs and expectations from the Wasfaty service were assessed, and its experience, concerns, and limitations were evaluated.

Methods: Qualitative, in-depth, semistructured interviews of Saudi community members who visited community pharmacies with electronic prescriptions from $\mathrm{MOH}$ primary healthcare clinics were conducted. The data obtained from 18 participants were thematically organized and analyzed using NVivo software.

Results: Seven themes related to the new Wasfaty service were identified and divided into three categories: perception toward the transition in pharmaceutical care, experience of the Wasfaty service, and concerns about and limitations of the Wasfaty service. The Saudi community was generally satisfied with the new Wasfaty service and highlighted its benefits: easier access, time flexibility, and less crowded community pharmacies. In addition, the Wasfaty service provided them with better communication with pharmacists, better education about their medications, better availability of medications, better control over medication dispensing, and easier refills compared to primary healthcare pharmacies. However, the community complained about the lack of privacy in community pharmacies, the number and location of community pharmacies having the Wasfaty service, a few technical issues, a lack of female pharmacists, few Saudi pharmacists, a lack of labels on medications, and limited or no instructions about medication storage.

Conclusion: The Saudi community needs the transition from MOH PHCs to the Wasfaty service in pharmaceutical care. The community also has concerns about this transition and the limitations of the Wasfaty service.

\section{Background}

A community pharmacy is defined as "a healthcare facility that is responsible for the provision of pharmaceutical services to a specific community."1 A community pharmacy allows the public access to medications and healthcare advice. In Saudi Arabia, there are $~ 9000$ community pharmacies under the regulation of the Ministry of Health $(\mathrm{MOH})$, the Saudi Food and Drug Authority (SFDA), and the Saudi Commission for Health Specialties (SCHS). The MOH Department of Pharmaceutical Care organizes and supervises pharmacy practice-related activities in Saudi Arabia, the SCHS is responsible for accreditation of pharmacists, and the SFDA ensures the safety of food, medications, biological and chemical substances, and electronic products. ${ }^{2,3}$ 
In 2016, Saudi Arabia launched its new Vision 2030 with a 15-year strategic plan. ${ }^{4}$ The $\mathrm{MOH}$ released its strategic plan for pharmaceutical care and the quantity and quality of national pharmacy practice programs as one of its 15 strategies to achieve Vision 2030. ${ }^{4}$ Under this plan, most of the pharmaceutical care services (e.g., dispensing, educating, monitoring therapy outcomes, solving drug therapy-related problems, and making drug therapy-related recommendations) would be provided through community pharmacies. ${ }^{4}$ However, the pharmaceutical care services provided in the Saudi community pharmacies are limited. ${ }^{5}$ In addition, the Saudi community has varying levels of satisfaction with the pharmaceutical care services, despite the good perception about the role of community pharmacists. ${ }^{6-8}$ In a recent study, community pharmacists indicated that poor facilities as well as a lack of supporting staff, public trust, clear regulations, and access to patient medical records are the main barriers to expanding their roles in community pharmacies. ${ }^{9}$

In 2018, the $\mathrm{MOH}$ transformed pharmaceutical care services from primary healthcare centers (PHCs) to private community pharmacies in order to meet Vision 2030. One of the goals of the MOH's strategic plan is to provide complete pharmacy electronic services in government and private healthcare settings. ${ }^{10}$ To achieve this goal, one of the projects implemented by the $\mathrm{MOH}$ was Wasfaty, ${ }^{11}$ an electronic system that enables physicians in the $\mathrm{MOH}$ primary care clinics to electronically initiate prescriptions for patients and send them directly to community pharmacies. Once a prescription is initiated, the patient receives a message including the patient ID and prescription code on the cell phone. Then, the patient can go directly to the nearest community pharmacy and collect medications. ${ }^{11}$ The Wasfaty program aims to implement the highest standards in order to facilitate medication-dispensing services, ensure availability of medications to all beneficiaries, and save time and resources for government health agencies. ${ }^{11}$

There is a lack of studies on the impact of the transition of pharmaceutical care services from PHCs to community pharmacies in Saudi Arabia. Therefore, this study explored the perception of the Saudi community (PHC patients) who benefit from this new Wasfaty service.

\section{Methods}

On the basis of the exploratory nature of this study, qualitative methods were deemed appropriate. Adult's members ( $>=18$ years old) of the Saudi community who visited community pharmacies in Riyadh, Saudi Arabia, with Wasfaty prescriptions from MOH PHCs (Wasfaty consumers) from February to March 2020 were invited to participate in the study. Those who agreed to participate were asked to sign a consent form. Once consent was obtained, the time and venue of the interview were fixed.

Semistructured, in-depth interviews were conducted for the convenience-based sample of Wasfaty consumers. The interviews were conducted in the quietest area (convenient to the interviewee) inside community pharmacies, and each interview took around 30-60 min to complete. Due to time limitations for a few participants, phone interviews were conducted either to complete the interviews or to conduct 
them after taking the participants' consent and the participants' contact number in the community pharmacy.

The sample size was not numerically defined, and interviews were conducted until data saturation, defined as no additional data being found by the researcher. ${ }^{12}$ Data saturation was independently determined by two members of the research team, and a third member ensured that the final few interviews yielded no novel information.

Data confidentiality was maintained by assigning a code to each participant and the interview transcript.

The semistructured interview guide was based on a literature review. It comprised four main parts: (i) demographics, (ii) perception toward the transition of pharmaceutical care services, (iii) experience with the new Wasfaty service and comparison with the old pharmaceutical care services provided in PHCs, and (iv) concerns and limitations. The research tool was evaluated by a group of researchers, and adjustments were made accordingly.

Next, the 20 audio-recorded interviews were transcribed verbatim and then translated into English by the main researcher. Data from 18 of the 20 interviews were analyzed; the data of 2 participants were excluded, as they were a pharmacist and a physician who worked in PHCs. The transcripts and their translation were independently checked for accuracy by two other researchers. Data in the translated transcripts were thematically organized and analyzed using NVivo software (QSR International, Doncaster, Australia). The transcripts were coded by two independent coders. Discussions were conducted to resolve any coding discrepancies and maintain consistency. Data validity was ensured through triangulation/peer debriefing with a superimposed researcher.

\section{Results}

Table 1 lists the demographics of the 18 participants. Seven themes related to the new Wasfaty service were identified and divided into three categories: perception toward the transition of pharmaceutical care services, experience of the new Wasfaty service, and concerns about and limitations of the Wasfaty service. Figure 1 shows the thematic concept map used in the study. 
Table 1

Sociodemographic characteristics of respondents $(n=18)$

\begin{tabular}{|ll|}
\hline Characteristics Number (\%) \\
\hline Gender & $13(72.2)$ \\
\hline Male & $5(27.8)$ \\
\hline Female & \\
\hline Yes & 0 \\
\hline No & $18(100)$ \\
\hline Age & \\
\hline $26-35$ & $3(16.7)$ \\
\hline $36-45$ & $6(33.3)$ \\
\hline $46-55$ & $6(33.3)$ \\
\hline $56-65$ & $3(16.7)$ \\
\hline Level of education & \\
\hline University degree & $9(50)$ \\
\hline Diploma & $1(5.6)$ \\
\hline High School & $5(27.8)$ \\
\hline Some high school or less & $3(16.7)$ \\
\hline Work status & \\
\hline Working (full time) & $11(61.1)$ \\
\hline Working (part time) & $2(11.1)$ \\
\hline Seeking work & $1(5.6)$ \\
\hline Not working & $4(22.2)$ \\
\hline Nationality & \\
\hline Saudi & \\
\hline Resident & \\
\hline Health insurance & \\
\hline Yes & \\
\hline
\end{tabular}




\begin{tabular}{|ll|}
\hline \multicolumn{2}{|l|}{ Characteristics Number (\%) } \\
\hline No & $16(88.9)$ \\
\hline
\end{tabular}

\section{Perception Toward The Transition In Pharmaceutical Care Theme: the need for the new Wasfaty service}

The majority of participants expressed a need for the new Wasfaty service and said that it is more convenient than PHCs for the following reasons: easy access, easy availability, and locations near homes.

"Yes, because there are plenty of community pharmacies near my house, which is very convenient when it comes to the medicine dispensing." (Participant \#2)

Community pharmacies open for longer hours compared to PHC pharmacies, and a few participants highlighted this time flexibility.

"Community pharmacies are open 24 hours, unlike PHC pharmacies, which have specific working hours, and medicines are dispensed on certain times" (Participant \#20)

A few participants said that community pharmacies save time because they are less crowded and the workload of community pharmacists is less compared to PHC pharmacists. Other participants believed that community pharmacists have a greater workload than PHC pharmacists as they cover both pharmacy consumers and Wasfaty patients, but that they provide better pharmaceutical care compared to PHC pharmacists.

"Yes. To avoid waiting, I think getting prescribed medicine from a nearby community pharmacy would be more convenient to me, and it would spare me long times of waiting" (Participant \#4)

"Surely it is fast. I waste a lot of time there (meaning PHCs Pharmacies) waiting in the crowding, and it takes a long time to get the medication. On the contrary, now, it is faster and saves time" (Participant \#9)

"No, I think community pharmacists have more workload because they cover Wasfaty patients in addition to the pharmacy's regular customers. However, community pharmacists explain more than PHC pharmacists do" (Participant \# 20)

The majority of participants said that communication with community pharmacists is better compared to PHC pharmacists. A few participants said that community pharmacists spend more time with them and provide them with more information about their medication. A few participants said the reason was the less workload they believe community pharmacists have, others felt it was the commercial nature of the 
community pharmacists' work, while most of the community pharmacists believed the reason to be a lack of physical barriers in community pharmacies that improves pharmacist-patient communication.

"When dispensing medicine in community pharmacies, communication with pharmacists is much better compared to the old dispensing method at the PHCs, because there are no barriers between me and the pharmacist. Besides, pharmacists in community pharmacies have plenty of time to explain how to use the medicine" (Participant \#1)

"Yes, it'll help improve pharmaceutical care services; community pharmacists have more time to communicate with and educate patients compared to those who work in the PHCs under the Ministry of Health" (Participant \#3)

"Unlike PHC pharmacists, community pharmacists are more communicative with patients; maybe because they are salesmen and the PHC pharmacist is a government employee. Community pharmacists explain medicines more than PHC pharmacists do. The latter explain only what the doctor has written without any further explanations or details" (Participant \#2)

A few participants indicated better availability of medications in community pharmacies compared to PHC pharmacies. In addition, the Wasfaty service is not limited to one pharmacy; if medications are not available in one community pharmacy, patients can find them in another one. One participant believed that community pharmacies provide better medication quality compared to $\mathrm{PHC}$ pharmacies.

"It contains medicines that are not available in the health centers" (Participant \#13)

"It is a beautiful institutional step, and societies desperately need it for several reasons. Perhaps the most important reason is the availability of medication in community pharmacies compared to PHC pharmacies" (Participant \#14)

"In the past, the medications that were being dispensed to us from PHCs were of little help or were of lesser quality. Some are made in .... or somewhere else. I'm not sure where. But when we began to fill our prescriptions from community pharmacies, we began to receive good quality medicines compared to PHCs. For example, I take insulin injections, and they are the best. I also take cholesterol medicine: I take everything. And these were not being dispensed to us by the PHCs" (Participant \#15)

"In PHCs not all medications were available in the pharmacy. They asked me to buy many medications from outside pharmacies" (Participant \#16)

Two of the participants believed that community pharmacies have more control over medication dispensing compared to PHC pharmacies. Two other participants said that the new Wasfaty service made medication refill easier because they did not need to book an appointment with PHC physicians for refill prescriptions. 
"Yes, because it is an opportunity to benefit from commercial or community pharmacies where medicine dispensing is controlled and legalized, unlike PHC pharmacies before when this process was random" (Participant \#2)

"There would be more control over medication dispensing" (Participant \#17)

"Well, it is better for us because instead of booking appointments every month in order to get the medication, we just bring this paper [prescription] and receive the medication. It is actually more comfortable for us" (Participant \#9)

"It is punctual here. Every 23rd of the month I come and take my medication. If there is something that is not available, I might come after a week or two. No problem." (Participant \#8)

\section{Theme: expectations from the new Wasfaty service}

The majority of participants did not believe that this transition would affect patients receiving their medications, while some believed that it would limit the patients from receiving their medications, because they would have to bear the cost of transportation.

"I don't think it will affect how patients receive medication because community pharmacies are available everywhere and are open all the time. I, therefore, can go there at any time to have my medication dispensed for me" ( Participant \#2)

"No, if the person is older or disabled and cannot go to the community pharmacy, they would probably have a son or driver to go instead. Now the person can go to the community pharmacy after an hour or two or 6 and find their medicines ready, and they would be able to fill the prescription immediately. And if they did not find the medicines at one pharmacy, they can find them at another" (Participant \#15)

"Yes. This will limit how the patients take and collect their medication from community pharmacies because it will add to the cost in terms of transportation and the time required to go there to collect the medicine. Moreover, Wasfaty program is not found at all community pharmacies" (Participant \#19)

The majority of participants expected to receive better pharmaceutical care in community pharmacies compared to PHC pharmacies. The main reasons were the availability of medications, communication with community pharmacists, and long opening hours of community pharmacies.

"Yes, I expect to receive better care because it is easy to communicate with the community pharmacist who has more time to educate and less work than the pharmacists in PHCs" (Participant \#1)

"Yes, it is already providing better services. It is better because every month I can get my medication within 5 minutes. On the other hand, at the PHC, I used to pay several visits; they would say, 'Come back later; the medication car has not arrived. Come next week, come the following week.' So, I pay for my medication, but I cannot afford it as my salary can't cover it" ( Participant \#8) 
"Yes. Here in PHC, the pharmacy was open until 3:00 pm or before Assr. Now the service is available until 10:00 pm, and there is no need for the PHC pharmacy, as I can get the medication I want from the pharmacies outside. So, that is much better for sure than before" (Participant \#12)

\section{Experience Of The Wasfaty Service \\ Theme: communication with pharmacists}

The majority of participants said that the Wasfaty service provides them with better communication with community pharmacists compared to PHC pharmacists. The participants complained of physical barriers in PHC pharmacies, which limited their communication with pharmacists.

"I can ask if I need to know about something, but at the PHC before, I couldn't ask because there wasn't any kind of communication" (Participant \#12)

"The pharmacy in the health center was fully covered, I couldn't see who's behind that covered wall if it was a man or a woman. I felt it was a problem but compared to the outside pharmacy I can talk freely with the pharmacist" (Participant \#10)

"I remember a long time ago, the pharmacist (in PHCS) would talk to you as if he were doing you a favor, not... The curtain is pulled down, the window is like you're inside a bank as if he is giving you money, dispensing money, not medication" (Participant \#17)

"In PHCs, there is a small barrier probably to reduces communication with the other gender. Sometimes it prevents a person from asking important but embarrassing questions, like applying ointments in private areas. A patient might not ask even if he needs the answer. It is embarrassing for some people. The barrier reduces communication between the patient and the pharmacist" (Participant \#14)

\section{Theme: medication education}

The majority of participants said that community pharmacists provide them with better education about their medications. A few participants said that there was no difference between community and PHC pharmacies in providing major instructions about medications, such as the dose, duration, and time to take medication (before/after meals or morning/bedtime). A few participants said they received no education or instructions in PHCs about their medications, and medication instructions were only written on the medications. In addition, a few participants said that they usually depended on their physicians for medication education.

"Community pharmacies provide more information than PHC pharmacies. I mean, they even write on it (mean medicine) how many times and so on, but they don't do that there. They just give me the medication, and sometimes I have to write it down by myself to remember. He doesn't care that much about these details. For instance, he says, 'take once a day as needed.' Most of their medication is taken 
as needed; how do I know when it is needed? If the doctor prescribed it for me, how come it is as needed?!! Most of the medications, he saied to be taken as needed' (Participant \#16)

"You might not receive complete information from the pharmacist at PHCs. He would write instructions on the package and you leave. But you are able to interact with the pharmacist at the community pharmacy and ask "how is this done?" and "if this or that..." no problem. The doctor at the PHCs is the one who explains the medicines, such as "take $10 \mathrm{ml}$ of this medicine three times a day for a week or 10 days," or for example "take these injections then we will do some laboratory tests, and depending on the results we will increase or decrease the dose." All of this is explained by the doctor, not the pharmacist. The pharmacist only gives you basic points when the patient needs them. But if the patient does not need them, then the doctor's instructions are enough. (Participant \#15)

A few participants believed that shortage of medications in PHCs and poor communication with PHC pharmacists could limit the medication education provided to them by PHC pharmacists. In addition, a few participants also believed that regulations over community pharmacists and the commercial nature of the job could be the reasons for better medication education provided by community pharmacists.

"The community pharmacist answered my concerns regarding medical indications, contraindications, and side effects. However, due to difficulty of communication, I could not pass any inquiries to the PHC pharmacist" (Participant \#1)

"Maybe the community pharmacy has all medicines available and the PHCs pharmacy only a limited range. This means that the community pharmacist is knowledgeable about drug companies and the prices and effects of medicines. They can also provide more than one option for medications with different prices" (Participant \#15)

"Community pharmacists are better in providing explanations about the medication because they deal with lots of medicines and lots of people, not only Wasfaty customers. On the other hand, the pharmacists' job at the PHCs is only to dispense medication, providing no explanations; i.e. his work is restricted to dispensing medication without providing explanations" (Participant \#20)

The majority of participants said that information about medication storage was never given to them, whether in PHCs or in community pharmacies, unless asked. A few participants said that only if a medication were a compounding preparation, they were informed to keep it in a cold place. The majority of participants also said both PHC and community pharmacists did not take the initiative to provide patients with additional information about their medications, unless asked, and a few participants complained that PHC pharmacists did not respond to their concerns about medications.

"There is very, very little information. Only in the case of compound solutions, they tell you to put it in a cold place" (Participant \#14)

"No, but sometimes they do, not always. For example, powdered antibiotics for children, they would tell me to fill it with water to the line then shake it. But they don't tell me what type of water to use, cold or 
warm or boiled" (Participant \#15)

"Both, the community pharmacist and the pharmacist at the PHC tell me how, when, and how long to take the medicine. They also explain to me any skills needed for taking the medicine. However, neither did they mention anything about storing the medicine or about the date of expiration after opening the package.

They need to be more careful about knowing the patients' medical history when explaining the medication; this applies to both the PHC and the community pharmacies. For example, I use Warfarin, and I need to know how to store it, contraindications, possible interactions with other medicines that I'm taking, food interaction with the medicines I'm taking, especially with Waferin, and the side effects. I want the pharmacist to tell me all this information without asking him, whether at the PHC or community pharmacies. The community pharmacist does not explain to me unless I ask him" (Participant \#19)

"No, they (PHCs pharmacists) usually tell you, ask the physician." (Participant \#14)

\section{Theme: medication dispensing}

All participants said that community pharmacies providing the Wasfaty service ask about the prescription reference number, ID number, or cell phone number and confirm the patient's name, while PHCs only handled written prescriptions and dispensed medications. A few participants also said that PHC pharmacists sometimes did not even confirm whether a patient was the correct one, and one participant said that he was afraid of being giving wrong medications.

"He checks the computer and finds my information. The process before was done by a written prescription that I dispense from the pharmacy. The medicine name might not be clear, there was always a fear that the pharmacist gives me another one, because it was handwritten, and the pharmacist might read it wrong. The system now is much better where they take your ID to ensure that you are the patient" (Participant \#10)

The majority of participants said that neither the PHC pharmacy nor the Wasfaty service at the community pharmacy put labels of instructions on their medications. Both community and PHC pharmacists wrote instructions on medication packages, indicating how many times a day the medication should be taken and whether in the morning or at bedtime or before/after meals.

"Pharmacists at PHC as well as community pharmacies do not put labels on the medicines. They only write information about when to take the medicine, before or after meals. They also draw lines to indicate the frequency of use, but they never mention anything about storage" (Participant \#2)

One participant believed that medications dispensed at community pharmacies are the best, as medications are given in their original packages, while another participant believed that the quantity of medications is much controlled under the Wasfaty service.

"No. Community pharmacies are the best in this concern. Each medication comes in a package with all the necessary information required by the Ministry of Health or Food and Drug Authority. PHCs, on the 
other hand, only give medication in the form of strips or in plastic bags which usually do not have labels. only have lines drawn on them" (Participant \#14)

"Medication before was dispensed in large quantities, but now, medication is dispensed in fewer quantities according to the patients' needs" (Participant \#19)

\section{Satisfaction, Concerns, And Limitations Of The New Wasfaty Service}

\section{Theme: satisfaction with the new Wasfaty service}

The majority of participants were satisfied with the new Wasfaty service. However, they had a few concerns and also mentioned a few limitations of the new service, related to the pharmacy, pharmacists, medications, or the system.

"In general, I am completely satisfied, as there was no delay in service. In addition, passing my inquiries about the medicine, educating and obtaining information was done with ease" (Participant \#1)

\section{Theme: concerns about and limitations of the new Wasfaty service}

The majority of participants complained about a lack of privacy in community pharmacies, because there is no private area for Wasfaty patients, although a few said that the condition is better compared to PHC pharmacies. In addition, a few participants highlighted the issue of a lack of female pharmacists in community pharmacies, and they believed that having female pharmacists would improve the privacy provided by the new Wasfaty service.

"No, there is no privacy, because medicine collection area in community pharmacies is open to all visitors" (Participant \#1)

"No. There is no privacy because the place is open. Having female pharmacists would be great, and it would provide privacy for us" (Participant \#19)

"Of course, if she was the pharmacist, l'd ask her things I couldn't ask before because it was a man" (Participant \#10)

"I think it is better now. I can deal with the pharmacist in a better way now; I can talk to him with a low voice. However, in the past, I had to speak loudly because of the window, and there could be people around, so it might be somehow embarrassing and less private. But now, the level of privacy is higher" (Participant \#12) 
"I get shy because sometimes I ask a question with a loud voice because he couldn't hear me (mean in PHCs pharmacy), and I don't like it when I have to raise my voice while everyone can hear me asking the pharmacist" (Participant \#10)

One concern a few participants raised was the location of community pharmacies, in addition to the number of community pharmacies providing the new Wasfaty service. A few participants suggested that there be a community pharmacy near or even inside each PHC. They believed this would help remove the cost of transportation and time and make it easier for them to get clarifications or alternative prescriptions from their physicians. In addition, a few participants said that some of their medications were not available at the first community pharmacy they visited, while others said that community pharmacists lack the authority to provide them with alternative medications if their medications are not available. These issues bothered them, in addition to the cost and time to get alternative prescriptions.

"This will limit how the patients take and collect their medication from community pharmacies because it will add to the cost in terms of transportation and the time required to go there to collect the medicine. Moreover, Wasfaty program is not found at all community pharmacies" (Participant \#19)

"To be honest, they are located a bit far away. I thought once they've closed the PHC pharmacies, they'll make sure there will be nearby pharmacies to everyone. Outside pharmacies have to be as close as possible to the PHC so that the patient can get his medication without walking all that distance" (Participant \#16)

"However, it should be more organized and of higher standards than it is now. For instance, the distribution of community pharmacies within neighborhoods should be better and more appropriate compared to their locations now. With all the traffic jams in Riyadh, reaching community pharmacies has become hard, in addition to the fact that they are located far from PHCs. This should be studied carefully. In other words, community pharmacies should be located near PHCs. Moreover, there should be enough parking lots that serve patients visiting the community pharmacies, because the less parking lots are found, the harder it would be to reach the pharmacies due to traffic jam. Sometimes, there is a shortage in medicine supply, which makes me visit several pharmacies in order to have my medicine prescribed. The good thing about community pharmacies is that they are available at any time. I suggest having a pharmacy close to the PHC in each neighborhood and connect it to the community pharmacies located on the main streets" (Participant \#20)

"I've noticed something strange; the doctors usually prescribe medications that aren't available in the pharmacies outside. There should be some kind of balance, or doctors should be updated with the medication database so that they would know if the medication is available outside the PHC or not. I have two prescriptions today; one is for my wife, and the other is for my daughter. The doctor prescribed a cream for my daughter, which I could not find although I've looked for it in several pharmacies. It was only last week. This is strange. How come that it is available in the doctor's database while it not available in the market outside the PHC' (Participant \#12) 
"However, when they moved [the service] to community pharmacies, firstly, pharmacies say that they're committed to a certain brand of medication, i.e. the same as what is written in the prescription. Alternatives are not allowed even if they are insulin injections; for instance, measurement injections. It would be great if they allow community pharmacies to provide alternatives. They could name the alternatives to the pharmacies, especially in the logistics which do not make a huge difference or do not include effective substance, such as widely-known diabetes drugs, injections, and swipe" (Participant \#14)

Another concern the participants had was technical issues with the Wasfaty service: either there were delays in community pharmacies receiving prescriptions, or patients did not receive prescription messages on their cell phones.

"When I take the medicine from the community pharmacy, the pharmacist said that he had to take it from the other computer (which takes time) and he asked me to come back after prayer time, and that delayed me because they have two systems: the regular dispensing system and the Wasfaty' (Participant \#10)

"No, I didn't receive an SMS. I've looked for it several times, but it didn't come. They told me l'd receive an SMS, but I didn't. The doctor only wrote the prescription for me" (Participant \#11)

Another issue raised by a few participants was "Saudization." They believed that Saudi pharmacists are more qualified and have communication skills that the community needs.

"Saudization of pharmacists in community pharmacies would give us more privacy and make us feel more comfortable because Saudi pharmacists at community pharmacies would be better in dealing with patients than the non-Saudi. No, I don't trust community pharmacists because they have poor communication skills, but they are more capable than PHC pharmacists. The national consensus is missing. Saudi pharmacists are compassionate towards Saudi patients, especially the elderly, more than non-Saudis do" (Participant \#20)

"Does the pharmacist in PHCs have more information and did he study better and understand better than the pharmacist at the community pharmacy? Only Allah knows. maybe the other (non-Saudi) studied only two years and cannot know all medications. He probably studied it as a trade to work in community pharmacy" (Participant \#15)

\section{Discussion}

This study is the first study to assess the Saudi community's perception of community pharmacies (the new Wasfaty service) with regard to needs, expectations, concerns, and limitations. In light of the proposed transformation of pharmacies in Saudi Arabia to achieve Vision 2030, the study provides a way to improve the Wasfaty service to meet the Saudi community's needs and satisfy their concerns. In addition, the study provides a conceptual framework to assess the long-term impact of the Wasfaty service. 
The Saudi community expressed its strong need for the transition of pharmaceutical care services from PHCs to community pharmacies and believes the new Wasfaty service will improve the process of receiving medications and provide them with better pharmaceutical care. Community pharmacies are considered more accessible, with extended operating hours and less crowds compared to PHC pharmacies. A recent study exploring the Saudi community's needs and preferences for community pharmacy immunization services also reported the same reasons. ${ }^{13}$ The Saudi community also believes that community pharmacists have better communication, spend more time with patients, and provide them with more information regarding their medications. These findings are consistent with a recent study that showed the Saudi community's positive perception and attitude toward community pharmacists in Saudi Arabia. ${ }^{8}$ In addition, the Saudi community believes that the new Wasfaty service will provide better availability of medications, better medication quality, and easier refills. A previous study reported issues of shortage and quality of medications provided at PHCs. ${ }^{14}$

A recent systematic review of 24 original studies conducted in Saudi Arabia showed that community pharmacists' knowledge and attitude are inadequate to provide quality patient care. ${ }^{15}$ The review showed that community pharmacists provide inadequate medication counseling, have insufficient knowledge about pharmacy laws and regulations in Saudi Arabia kingdom with regard to reporting adverse drug reactions (ADRs), and have insufficient knowledge about regulations for prescribing and dispensing medications as antibiotics. The review also highlighted the need for a training program for community pharmacists for them to be able to effectively deliver patient-centered care. ${ }^{15}$ Previous studies have also shown that the Saudi community is poorly satisfied with community pharmacists' performance as healthcare providers. ${ }^{6,7,16}$ However, in this study, the Saudi community was generally satisfied with the new Wasfaty service and indicated that the communication with community pharmacists was better compared to PHC pharmacists, community pharmacies better educate patients about their medications, and medications given in original packages are in good, required amounts. The one issue point that the Saudi community raised is that both community and PHC pharmacists do not take the initiative to provide patients with additional information about their medications unless asked, which is consistent with previous studies in which participants indicated that they need to be inquisitive for information. $7,16,17$

One of the key themes expressed by the Saudi community was concerns about and limitations of the new Wasfaty service, such as a lack of privacy, unavailability of female pharmacists, and a limited number of Saudi pharmacists, which is consistent with a recent study that explored the Saudi community's needs and preferences for immunization services in community pharmacies. ${ }^{13}$ It is likely that such concerns are valid and will affect adoption of the new Wasfaty service by the Saudi community. In fact, the MOH, in collaboration with the Ministry of Human Resources and Social Development (MHRSD), released an initiative targeting Saudization of the pharmacy profession in Saudi Arabia. ${ }^{18}$ Given the nature of gender sensitivity in Saudi culture, it is important to increase the number of female pharmacists working in Saudi community pharmacies. Addressing the privacy issue is also important because a lack of privacy in community pharmacies prevents community pharmacists from providing complete information and instructions about medication use to patients. ${ }^{19}$

Page $15 / 21$ 
Another concern the Saudi community had was a lack of labels on medications, with no or limited instructions provided by both community and $\mathrm{PHC}$ pharmacists with regard to proper medication storage. These concerns are consistent with previous studies on PHCs. ${ }^{14,17}$ In fact, proper medication labeling and counseling is one of the responsibilities assigned to community pharmacists providing the Wasfaty service. However, the $\mathrm{MOH}$ and SFSA should provide clear regulations for what is expected of community pharmacists during the dispensing of medications, as the Saudi Executives Roles for Institutional and Pharmaceutical Products law does not cover this aspect. ${ }^{20}$ ASHP guidelines on pharmacists conducting patient education and counseling include storage of medication. ${ }^{21}$ Training community pharmacists working with the Wasfaty service on good communication skills, ideal counseling, and good medicationdispensing methods would be helpful in addressing these limitations of the Wasfaty service.

The Saudi community is also concerned about the number and location of community pharmacies providing the Wasfaty service. Currently, the number of community pharmacies providing the Wasfaty service around Saudi Arabia is 1588 community pharmacies within 23 pharmacy chains. ${ }^{11}$ Given the plan is to provide the Wasfaty service in $100 \%$ of all PHCs and governmental hospitals by 2021 , the $\mathrm{MOH}$ should consider the Saudi community's need for increasing the number of community pharmacies covering Wasfaty patients. In addition, the $\mathrm{MOH}$ should consider opening community pharmacies providing the Wasfaty service inside or near each PHC to avoid the cost and time spent on transportation by patients.

This study had a few limitations. First, the results cannot be externally generalized, because the study participants were only from Riyadh. However, data generalizability was not the intention; instead, the goal was to ascertain the Saudi community's needs, explore its perceptions toward the transition of pharmaceutical care from PHCs to community pharmacies, and explore its perceptions toward the limitations of the new Wasfaty service. Second, the interviews were conducted in Arabic (the community language) and then translated into English to be analyzed by NVivio. NVivo requires transcripts to be in English, which means the meaning of particular words might have been lost during translation. ${ }^{22,23}$ To avoid this, a back-translation of a few randomly selected interviews was done by a different researcher from those who conducted the interviews.

\section{Conclusion}

This study assessed the Saudi community's perception toward the transition of pharmaceutical care services from PHCs to community pharmacies with regard to needs, expectations, satisfaction, concerns, and limitations. The Saudi community needs this transition and is generally satisfied with the new Wasfaty service. Transition of pharmaceutical care from PHCs to community pharmacies is a good step to improving the pharmaceutical care provided to $\mathrm{MOH}$ patients. The findings can be used to improve the current implementation of the Wasfaty service to meet the Saudi community's needs and satisfy their concerns. 


\section{Recommendations}

On the basis of our results, here are a few recommendations that could help in improving the new Wasfaty service:

1. The Wasfaty service should be implemented across the country, and the number of community pharmacies providing this service should be increased.

2. Community pharmacists should be provided with training on good communication skills, ideal counseling, and good medication-dispensing methods.

3. The MOH should reconsider the Saudi community's need for opening community pharmacies providing the Wasfaty service inside or near each $\mathrm{PHC}$ to avoid the cost and time spent on transportation by patients.

4. Some of the regulations with regard to authorities community pharmacies providing the Wasfaty service have should be adjusted, if required, such as the authority to provide alternative medications.

5. On the basis of the Saudi community's needs and cultural sensitivity, Saudization of community pharmacies and employment of female pharmacists need to be addressed by the $\mathrm{MOH}$.

6. The new Wasfaty service should be periodically assessed.

\section{Recommendations for future research:}

- The community pharmacies' perception toward the transition in pharmaceutical care from PHCs to community pharmacies needs to be assessed in order to evaluate the new Wasfaty service from a different perspective.

- Future studies should assess the economic impact of this transition in pharmaceutical care, including the impact on patients and the $\mathrm{MOH}$ (both direct and indirect costs).

- Implementing the study in different regions around Saudi Arabia will help assess different community concerns about and limitations of the new Wasfaty service.

\section{Abbreviations}

ADR, adverse drug reaction; IRB, institutional review board; MHRSD, Ministry of Human Resources and Social Development; $\mathrm{MOH}$, Ministry of Health; PHC, primary healthcare center; SCHS, Saudi Commission for Health Specialties; SFDA, Saudi Food and Drug Authority

\section{Declarations}

\section{Ethics approval and consent to participate}

The study was conducted in compliance with the ethical guidelines of the institutional review board (IRB) of King Saud University (KSU-IRB 017E) and the ethical guidelines of the IRB of the Riyadh Elm University (FPGRP/2019/437), and was approved by both IRBs. 


\section{Consent for publication}

All participant accepted to participate asked to sign a consent form highlighted that that personal information collected over the course of this project will be stored securely and will only be used for purposes that participant agreed to. Moreover, the results of this study will be published, and that publications will not contain any participant name or any identifiable information about participants.

\section{Availability of data and materials}

The datasets used and/or analyzed during the current study are available from the corresponding author on reasonable request.

\section{Competing Interest}

The authors declare that they have no known competing financial interests or personal relationships that could have appeared to influence the work reported in this paper.

\section{Acknowledgements and Funding}

The authors would like to acknowledge all of the participants in this study. The authors extend their appreciation to the Deanship of Scientific Research at King Saud University for financial support of this work, and to the RSSU at King Saud University for their technical support.

\section{Authors' contributions}

All authors substantially contributed to the conception and design of the study. SA conducted the interviews, transcribed the Audio-recorded interviews and then translated into English. NA checked transcripts and their translation for accuracy. MW doubled checked accuracy by conducting a back translation of some randomly choose interviews. SA and NA coded the transcripts independently and analyzed the data. TA was act as a peer debriefing. All authors have also participated in drafting and revising the manuscript and approved of the final version.

\section{References}

1. Saini R. Introduction. In: A textbook of community pharmacy. New Delhi: New Age International Publishers; 2012.

2. SFDA. About. SFDA [Internet]. [cited 2020 Sep 25]. Available from: https://www.sfda.gov.sa/en/overview.

3. Almeman A, Al-jedai A. Pharmacy practice in the Kingdom of Saudi Arabia. In: Pharmacy Practice in Developing Countries. Elsevier Inc.; 2015:171-97.

4. Alomi YA. New pharmacy model for vision 2030 in Saudi Arabia. J Pharm Pract Community Med [Internet]. 2017;3 (3https://www.ncbi.nlm.nih.gov/pmc/articles/PMC3917174/):194-6. Available from: http://www.jppcm.org/archives/sites/default/files/10.5530jppcm.2017.3.40.pdf. 
5. Alanazi AS, Alfadl AA, Hussain AS. Pharmaceutical Care in the Community Pharmacies of Saudi Arabia: Present Status and Possibilities for Improvement Pharmaceutical care Health care system in Saudi Arabia. Saudi J Med Med Sci [Internet]. 2016;(4):9-14. Available from: http://www.sjmms.net/temp/SaudiJMedMedSci419-2500109_065641.pdf.

6. Tannir M, Al, Alharbi Al, Alfawaz AS, Zahran RI, Altannir M. Saudi adults satisfaction with community pharmacy services. Springerplus [Internet]. Springer International Publishing; 2016;(5):1-5. Available from: https://www.ncbi.nlm.nih.gov/pmc/articles/PMC4912500/pdf/40064_2016_Article_2442.pdf.

7. Al-arifi MN. Patients' perception, views and satisfaction with pharmacists ' role as health care provider in community pharmacy setting at Riyadh, Saudi Arabia. Saudi Pharm J [Internet]. King Saud University; 2012;20(4):323-30. Available from: http://dx.doi.org/10.1016/j.jsps.2012.05.007.

8. Aloshaywi M. The public perception and attitude toward community pharmacists in Saudi Arabia. In: College of Pharmacy Research Day [Internet]. 2019. p. 22. Available from: https://fac.ksu.edu.sa/sites/default/files/9th_coprd_abstract_booklet_2019-final.pdf.

9. Alkhalaf $\mathrm{H}$, Alturki H. Saud pharmacist perception of their current role, Barriers and facilitator of expanded pharmacist roles in community pharmacy practice setting. In: College of Pharmacy Research Day [Internet]. 2019. p. 26. Available from: https://fac.ksu.edu.sa/sites/default/files/9th_coprd_abstract_booklet_2019-final.pdf.

10. Ahmed Y, Pharm A, Pharm C. National Pharmacy Practice Programs at Ministry of Health in Saudi Arabia Journal of Pharma and Pharmaceutical Sciences National Pharmacy Practice Programs at Ministry of Health in Saudi Arabia. J Pharma Pharm Sci. 2015;1(2):17-8.

11. Wasfaty. About Wasfaty [Internet]. [cited 2020 Sep 26]. Available from: https://wasfaty.sa/about/.

12. Gibbs G. Analyzing Qualitative Data. 2nd ed. SAGE Publications Ltd; 2018. 232 p.

13. Aloola N, Al, Alsaif R, Alhabib H, Alhossan A. Community needs and preferences for community pharmacy immunization services. Vaccine [Internet]. Elsevier Ltd; 2020;38(32):5009-14. Available from: https://doi.org/10.1016/j.vaccine.2020.05.060.

14. Alomi YA. Patient satisfaction of pharmaceutical care system at Ministry of Health in Saudi Arabia. BAOJ Pharm Sci. 2016;2(4):2-6.

15. Rasheed MK, Shahzad HS, Babar Z. Community pharmacists knowledge, attitude, roles, and practices patient-centred care in Saudi Arabia: A Systematic Review of the Literature. J Pharm Heal Serv Res [Internet]. 2018;10(1):101-15. Available from:

https://pure.hud.ac.uk/en/publications/community-pharmacists-knowledge-attitude-roles-andpractices-towa.

16. Alotaibi HS, Abdelkarim MA. Consumers ' perceptions on the contribution of community pharmacists in the dispensing process at Dawadmi. Saudi Pharm J [Internet]. King Saud University; 2015;23(3):230-4. Available from: http://dx.doi.org/10.1016/j.jsps.2014.11.002.

17. Alaqeel S, Abanmy NO. Counselling practices in community pharmacies in Riyadh, Saudi Arabia : a cross-sectional study. BMC Health Serv Res [Internet]. BMC Health Services Research; 2015;15:1-9. Available from: http://dx.doi.org/10.1186/s12913-015-1220-6. 


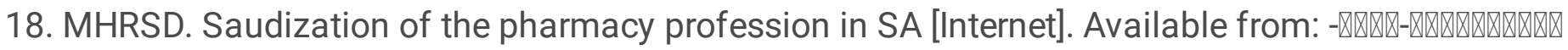

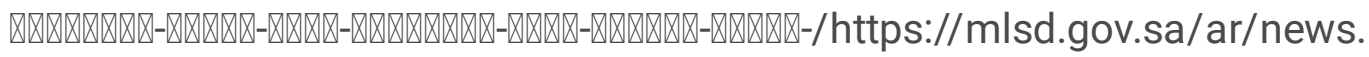

19. Bawazir SA. Consumer attitudes towards community pharmacy services in Saudi Arabia. Int J Pharm Pract. 2004;12(2):83-9.

20. SFDA. Executive roles for institutions and pharmaceutical products law [Internet]. Available from: http://www.sfda.gov.sa/AR/DRUG/DRUG_REG/ \%0D\%0APages/drug_reg.aspx. Accessed August 2020\%0D\%0A.

21. ASHP. ASHP Guidelines on Pharmacist-Conducted Patient Education and Counseling [Internet]. 1997. Available from: https://www.ashp.org/-/media/assets/policyguidelines/docs/guidelines/pharmacist-conducted-patient-education-counseling.ashx.

22. Chen $\mathrm{H}$, Boore JRP. Translation and back-translation in qualitative nursing research: methodological review. J Clin Nurs [Internet]. 2009;886(193):234-9. Available from: http://pdfs3.xuebalib.com:1262/xuebalib.com.14326.pdf.

23. Nes $F$, Van, Abma TA, Jonsson $H$, Institutet $K$, Deeg DJH. Language differences in qualitative research: Is meaning lost in translation? Eur J Ageing. 2010;7:313-6.

\section{Figures}



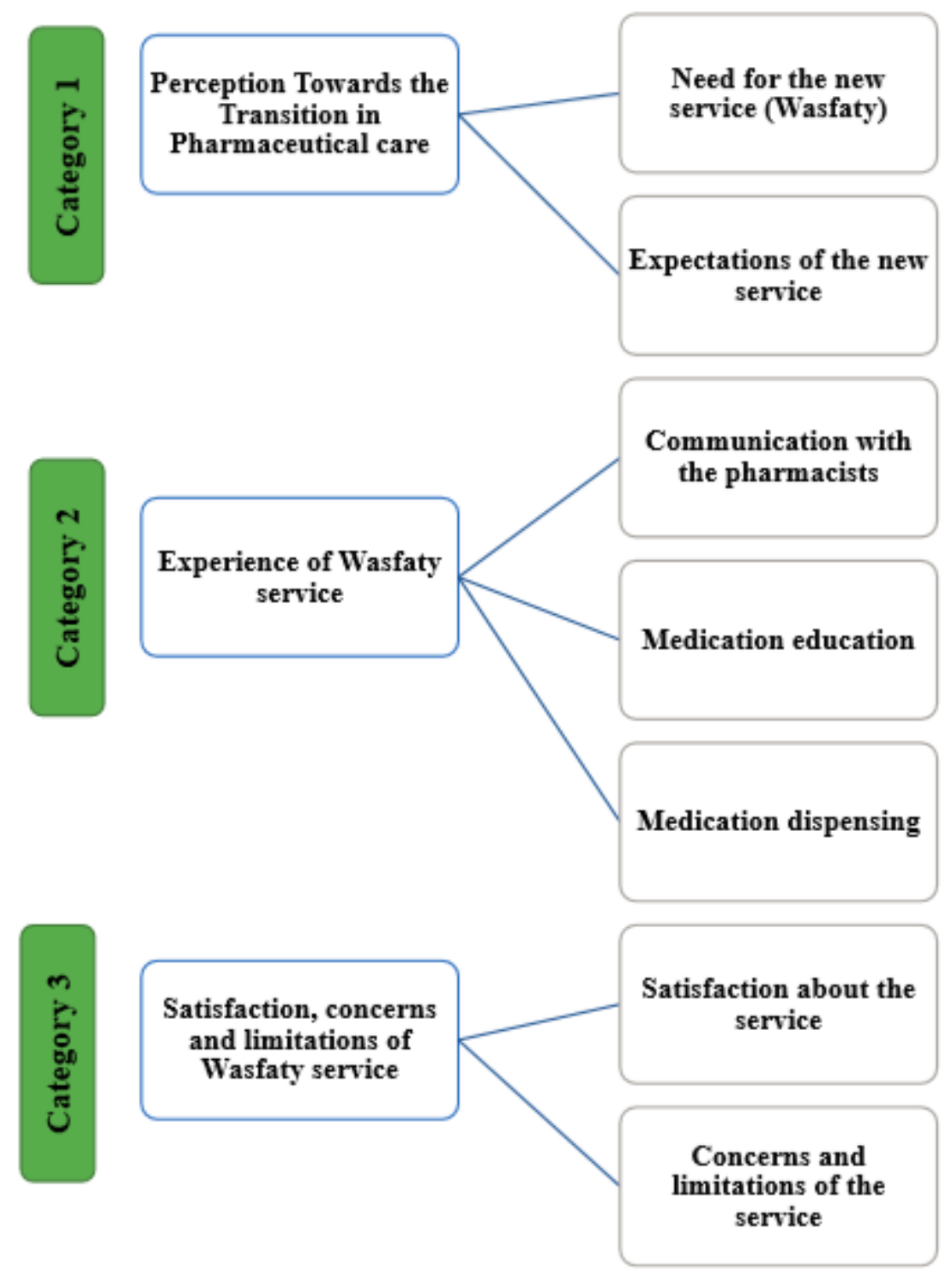

Figure 1

Thematic concept map used in the study

\section{Supplementary Files}

This is a list of supplementary files associated with this preprint. Click to download.

- interviewguide.pdf 